\title{
Resonant pairing between Fermions with unequal masses
}

\author{
Shin-Tza Wu and C.-H. Pao \\ Department of Physics, National Chung Cheng University, Chiayi 621, Taiwan \\ S.-K. Yip \\ Institute of Physics, Academia Sinica, Nankang, Taipei 115, Taiwan
}

(Dated: October 28, 2018)

\begin{abstract}
We study via mean-field theory the pairing between Fermions of different masses, especially at the unitary limit. At equal populations, the thermodynamic properties are identical with the equal mass case provided an appropriate rescaling is made. At unequal populations, for sufficiently light majority species, the system does not phase separate. For sufficiently heavy majority species, the phase separated normal phase have a density larger than that of the superfluid. For atoms in harmonic traps, the density profiles for unequal mass Fermions can be drastically different from their equal-mass counterparts.

PACS numbers: 03.75.Ss, 05.30.Fk, 34.90.+q
\end{abstract}


Cooper pairing between Fermions due to the presence of an attractive interaction is the essential physics for most, if not all, superconductors. In recent years, Feshbach resonances 1] provide us with a wonderful opportunity to further study strongly interacting Fermi systems. For wide resonances, the Feshbach resonance between the atoms can be viewed as providing an attractive pairing interaction, with the pairing strength increases from positive detuning towards negative detuning. For the case of equal populations of two Fermionic species and an s-wave interaction between them, it was predicted long time ago 2] that the system can smoothly crossover from a BCS superfluid to a Bose Einstein condensate of tightly bound pairs. Recent experiments have provided a confirmation of this picture, and many thermodynamical and dynamical properties have been measured. [3] There is also an explosion of theoretical activities studying this system. [4]

In the case where the populations of the Fermions are unequal, the smooth crossover discussed above is known to be destroyed [5]. Recent experiments [6] have shown that phase separation occurs near resonance, so that the superfluid appears near the center of the trap and a normal fluid of unequal population density appears outside the superfluid core. [7]

In the literature cited above, the Fermi atoms involved are identical except their internal electronic states. Hence they have identical masses. However, Feshbach resonances are not confined to the same atom species [8]. This thus raises the possibility of studying Fermion pairing between two atomic species of very different masses, with either equal or unequal populations. This problem can also have important implications in other areas of physics such as quark matter 9] and superconductivity in general.

In this paper, we thus study two Fermionic species of unequal masses with an interspecies short-ranged s-wave attractive interaction. First we consider the equal population case. We show that, at zero temperature and within mean-field theory [2], the thermodynamic properties of this system have a one-to-one correspondence with those of the equal mass case, provided we rescale the energies appropriately. Next we consider the case of unequal population, but confining ourselves to the unitary regime where the scattering length diverges. Defining $\gamma$ as the ratio of the majority atom mass to the minority mass, we show that the system phase separates for $\gamma>\gamma_{H} \approx 0.13$ but remains homogeneous for $\gamma<\gamma_{H}$. For sufficiently light majority particles, it is energetically costly to phase separate out the majority particles since a high Fermi energy of the majority species will result. For $\gamma>\gamma_{H}$, we show that the system phase separates into a superfluid with equal population density and a nor- 
mal fluid with unequal population density. However, there is dramatic difference between the cases where $\gamma$ is larger or less than a value $\gamma_{*} \approx 3.9$. For $\gamma_{H}<\gamma<\gamma_{*}$, the density of the normal fluid is less than the superfluid, as one may expect due to the attractive interaction. However, for $\gamma>\gamma_{*}$, the normal fluid is actually denser than the superfluid. We explain this by the larger density of states for the heavy majority component. We illustrate this difference by calculating the density profile of an unequal population gas in an harmonic trap.

We shall then consider at zero temperature two Fermionic species with a short-ranged interaction characterized by the s-wave scattering length $a .1 / a$ increases from $-\infty$ to $+\infty$ with the strength of the pairing interaction. We shall call the two species up and down, with masses $m_{\sigma}, \sigma=\uparrow$ or $\downarrow$. The effective mean-field Hamiltonian is $H_{m f}-\mu_{\uparrow} N_{\uparrow}-\mu_{\downarrow} N_{\downarrow}=$ $\sum_{\vec{k}, \sigma} \xi_{\sigma}(k) c_{\vec{k}, \sigma}^{\dagger} c_{\vec{k}, \sigma}-\sum_{\vec{k}}\left[\Delta^{*} c_{-\vec{k}, \downarrow} c_{\vec{k}, \uparrow}+c . c\right]-\left[\frac{m_{r}}{2 \pi \hbar^{2} a}-\sum_{\vec{k}} \frac{2 m_{r}}{k^{2}}\right]|\Delta|^{2}$. Here $\xi_{\sigma}(k)=\hbar^{2} k^{2} / 2 m_{\sigma}-\mu_{\sigma}$ are the quasi-particle excitation energies for normal fermions relative to their respective chemical potentials $\mu_{\sigma}, c_{\vec{k}, \sigma}$ 's are the annihilation operators for the Fermion $\sigma$ at momentum $\vec{k}$, c.c. denotes the complex conjugate, $m_{r}$ is the reduced mass $\left(m_{r}^{-1}=m_{\uparrow}^{-1}+m_{\downarrow}^{-1}\right) . \Delta$, the off-diagonal pairing potential, is determined by minimization of the expectation value of this Hamiltonian (i.e. the free energy [10]) with respect to $\Delta$. This gives, for equal populations, (see, e.g [11])

$$
-\frac{m_{r}}{2 \pi \hbar^{2} a} \Delta=\Delta \frac{1}{V} \sum_{\vec{k}}\left[\frac{1}{E_{\uparrow}(k)+E_{\downarrow}(k)}-\frac{2 m_{r}}{\hbar^{2} k^{2}}\right] .
$$

$E_{\sigma}(k)$, the quasiparticle energies at wavevector $k$, are given by

$$
E_{\sigma}(k)=\frac{\xi_{\sigma}(k)-\xi_{-\sigma}(k)}{2}+\sqrt{\left(\frac{\xi_{\sigma}(k)+\xi_{-\sigma}(k)}{2}\right)^{2}+\Delta^{2}}
$$

where and $-\uparrow \equiv \downarrow$. Defining $\mu \equiv\left(\mu_{\uparrow}+\mu_{\downarrow}\right) / 2$ and $h \equiv\left(\mu_{\uparrow}-\mu_{\downarrow}\right) / 2$ and noticing that $\xi_{\sigma}(k)+\xi_{-\sigma}(k)=\frac{\hbar^{2} k^{2}}{2 m_{r}}-2 \mu$, one obtains

$$
E_{\uparrow}(k)+E_{\downarrow}(k)=2 \sqrt{\left(\frac{\hbar^{2} k^{2}}{4 m_{r}}-\mu\right)^{2}+|\Delta|^{2}} \equiv 2 \tilde{E}(k) .
$$

The number equation is

$$
n=\frac{1}{V} \sum_{k} v^{2}(k)
$$


where $v(k)$, the usual coherence factor, is given by [1]] $v^{2}(k)=\frac{E_{\uparrow}-\xi_{\uparrow}}{E_{\uparrow}+E_{\downarrow}}=\frac{E_{\downarrow}-\xi_{\downarrow}}{E_{\uparrow}+E_{\downarrow}}$ and hence

$$
v^{2}(k)=\frac{1}{2}\left(1-\frac{\hbar^{2} k^{2} / 4 m_{r}-\mu}{\sqrt{\left(\frac{\hbar^{2} k^{2}}{4 m_{r}}-\mu\right)^{2}+|\Delta|^{2}}}\right)
$$

The equations (11) and (4) determine $\Delta$ and $\mu$ for a given density. Using eqs (3) and (5), we see that these equations are identical to those [2] of equal masses $m_{\uparrow}=m_{\downarrow}=m$ provided we substitute $m \rightarrow 2 m_{r}$. Thus their solutions are identical after this replacement. (The solutions are thus independent of $h$ so long as all quasiparticle energies $E_{\sigma}(k)>0$ : This condition also guarantees that $n_{\uparrow}=n_{\downarrow}$, see below). Therefore their solutions can be expressed as

$$
\begin{aligned}
& \mu=\frac{\epsilon_{F r}}{2} \zeta_{1}\left(\frac{1}{k_{F} a}\right) \\
& \Delta=\frac{\epsilon_{F r}}{2} \zeta_{2}\left(\frac{1}{k_{F} a}\right)
\end{aligned}
$$

where the dimensionless functions $\zeta_{1,2}$ are identical to the corresponding ones for equal masses [2]. Here $\epsilon_{F r} \equiv k_{F}^{2} / 2 m_{r}$ and $k_{F} \equiv\left(3 \pi^{2} n\right)^{1 / 3}$, where $n$ is the total density. We have introduced a factor of $1 / 2$ in eqs (6) and (7) since $\epsilon_{F r} / 2=k_{F}^{2} /(2 m)$ if $m_{\sigma}=m$. It follows immediately that other thermodynamical properties can be obtained similarly. For example, the energy density $E_{S} / V$ can be written as $E_{S} / V=n \frac{\epsilon_{F r}}{2} \zeta_{3}\left(\frac{1}{k_{F} a}\right)$ where $\zeta_{3}$ is again the same function as the equal mass case.

In particular, at unitarity $(a=\infty)$, we have $\mu=\frac{\epsilon_{F r}}{2} \zeta_{1}(0)$ where $\zeta_{1}(0) \approx 0.59$ in the present theory (and thus $\mu>0$ ). The magnitude of the order parameter $\Delta=\frac{\epsilon_{F r}}{2} \zeta_{2}(0)$ with $\zeta_{2}(0) \approx 0.68$ and hence $\Delta / \mu \approx 1.16$. Using the fact that the only length scale at unitarity is $k_{F}^{-1}, \zeta_{3}(0)=\frac{3}{5} \zeta_{1}(0)$ and thus $E_{S} / V=\frac{3}{5} n \frac{\epsilon_{F r}}{2} \zeta_{1}(0)$. At chemical potential $\mu$, from eq (6), $k_{F}=\left[4 m_{r} \mu / \zeta_{1}(0)\right]^{1 / 2}$, hence the density is given by $n=\frac{1}{3 \pi^{2}}\left[4 m_{r} \mu / \zeta_{1}(0)\right]^{3 / 2}$. Since the free energy density is $\Omega_{S} / V=E_{S} / V-\mu n=-\frac{2}{5} n \mu$, therefore

$$
\frac{\Omega_{S}}{V}=-\frac{2}{15 \pi^{2}} \frac{\left(4 m_{r}\right)^{3 / 2} \mu^{5 / 2}}{\zeta_{1}^{3 / 2}(0)} .
$$

Mean field theory is known to produce a larger $\zeta_{1}(0)$ than Monte-Carlo and experiments $(\approx 0.44)$ for the equal mass case $[3,4]$. It is however of interest to see to what extent $\zeta_{1}$ etc will depend on $\gamma$ in the unequal mass case in more accurate calculations. 
Next we consider unequal populations but confine ourselves to unitarity $(1 / a=0)$. We shall call $\uparrow(\downarrow)$ the majority (minority) species. The self-consistent order parameter equation reads

$$
0=\frac{\Delta}{V} \sum_{\vec{k}}\left[\frac{1-f\left(E_{\uparrow}(k)\right)-f\left(E_{\downarrow}(k)\right)}{E_{\uparrow}(k)+E_{\downarrow}(k)}-\frac{2 m_{r}}{\hbar^{2} k^{2}}\right] .
$$

Due to the appearance of the Fermi functions $f$ with arguments $E_{\sigma}(k)$, the solution to this equation does not depend on the reduced mass alone. $\left(n_{\uparrow}>n_{\downarrow}\right.$ requires that some $E_{\uparrow}(k)$ 's are negative, since $n_{\uparrow}-n_{\downarrow}=\frac{1}{V} \sum_{\vec{k}} f\left(E_{\uparrow}\right)$, see, e.g. [5], 11] ). By rescaling all energies to $\mu$, it is easy to see that eq (9) gives $\Delta / \mu$ as a function of $h / \mu$ for each $\gamma \equiv m_{\uparrow} / m_{\downarrow}$. The non-trivial $(\Delta \neq 0)$ solutions to eq (9) consists of two branches (see inset of Fig (1). One branch has $\Delta / \mu \approx 1.16$ independent of $h / \mu$ and $\gamma$. This branch corresponds to $E_{\sigma}(k)>0$ for all $k$ and $\sigma$, i.e., no quasiparticles (and hence equal populations). This solution has already been described in the last paragraph above. Another branch, corresponding to $n_{d}=n_{\uparrow}-n_{\downarrow} \neq 0$, gives a $h / \mu$ and $\gamma$ dependent $\Delta / \mu$. For $\gamma<0.13, \Delta / \mu$ decreases with increasing $h / \mu$. We have verified that this branch is a free energy [10] minimum, and thus represents a stable homogeneous superfluid. (We have also verified that this state has only one Fermi surface, and hence it does not correspond to a breached-pair state [9]) However, for $\gamma>0.13, \Delta / \mu$ increases with increasing $h / \mu$ (and correspondingly, for some range of $h$, $\Delta / \mu$ is multi-valued). This branch is a generalization of the solution obtained by Sarma 12 in the BCS limit for $\gamma=1$. We found that this solution corresponds to a free energy 10 ] relative maximum and is thus unstable.

For a homogeneous system with $\gamma>0.13$, the likely scenario is that the system phase separates, as [6] in the case of $\gamma=1$. (We ignore the more exotic possibilities [13] here). To characterize this phase, we seek the point where the free energy density of the completely paired superfluid state is equal to that of the normal fluid, i.e., $\Omega_{S}(\mu, h) / V=\Omega_{N}(\mu, h) / V$. $\Omega_{S} / V$ is independent of $h$ and was already given before. Within our mean field theory, $\Omega_{N} / V$ is just the free energy density of an non-interacting Fermi gas, thus $\Omega_{N} / V=-\frac{1}{15 \pi^{2}}$ $\left[\left(2 m_{\uparrow}\right)^{3 / 2}(\mu+h)^{5 / 2}+\left(2 m_{\downarrow}\right)^{3 / 2}(\mu-h)^{5 / 2} \mathcal{H}\right]$ where $\mathcal{H}=1$ if $\mu-h>0$ and $=0$ otherwise. Equating the free energies, we obtain an equation for $h / \mu$. Denoting this value as $(h / \mu)_{c}$, 
we get (using $\mu>0$ at resonance)

$$
\frac{1}{\zeta_{1}^{3 / 2}(0)}=\frac{1}{2}\left[\left(\frac{1+\gamma}{2}\right)^{3 / 2}\left(1+\left(\frac{h}{\mu}\right)_{c}\right)^{5 / 2}+\left(\frac{1+\gamma^{-1}}{2}\right)^{3 / 2}\left(1-\left(\frac{h}{\mu}\right)_{c}\right)^{5 / 2} \mathcal{H}\right]
$$

For $h / \mu<(h / \mu)_{c}$, we have a paired superfluid, whereas for $h / \mu>(h / \mu)_{c}$, we have a normal fluid. At $h / \mu=(h / \mu)_{c}$, the system phase separates into a superfluid region and a normal region. The dependence of $(h / \mu)_{c}$ on $\gamma$ is given in the main part of Fig $1,(h / \mu)_{c}>1$ for small $\gamma$, decreases with increasing $\gamma$, equals unity at $\gamma=\gamma_{P}=\zeta_{1}^{-1}(0)-1 \approx 0.69$, changes sign at $\gamma=\gamma_{*} \approx 3.9$, and is negative for $\gamma>\gamma_{*}$. (One should not be alarmed by this sign change: the concentration of up particles in the normal state is larger than that of the down particles if $m_{\uparrow}(\mu+h)>m_{\downarrow}(\mu-h)$, i.e., $\frac{h}{\mu}>\frac{1-\gamma}{1+\gamma}$. $)$ This behavior is the result of unequal masses of the two species. This is particularly clear if we examine the behavior of $(h / \mu)_{c}$ for large $\gamma$. In this limit, the solution to eq (10) is given asymptotically by $(h / \mu)_{c}=-1+c_{1} / \gamma^{3 / 5}$ where $c_{1}=2\left[\left(\zeta_{1}(0)\right)^{-3 / 2}-1\right]^{2 / 5}$. The dependence on $\gamma$ is due to the different density of states between the two species. With $\zeta_{1}(0) \approx 0.59$, we find $c_{1} \approx 2.16$. This asymptotic is plotted also in Fig 1, which gives a reasonable description to the actual solution.

The ratio of the minority to majority density is $\left(n_{\downarrow} / n_{\uparrow}\right)_{N}=$ $\mathcal{H}\left[\left(1-(h / \mu)_{c}\right) /\left(\gamma\left(1+(h / \mu)_{c}\right)\right)\right]^{3 / 2} . \quad\left(n_{d} / n\right)_{N}$ can be obtained via $\left(1-\left(n_{\downarrow} / n_{\uparrow}\right)_{N}\right) /(1+$ $\left.\left(n_{\downarrow} / n_{\uparrow}\right)_{N}\right)$. This ratio as a function of $\gamma$ is plotted in Fig $2, \quad\left(n_{d} / n\right)_{N}=1$ for $\gamma<\gamma_{P}$. There the only stable normal phase is the completely polarized phase. Since $n_{\downarrow}=n_{\uparrow}$ in the superfluid phase, $\left(n_{d} / n\right)_{N}$ is also the maximum allowed $\left(n_{d} / n\right)$ for the phase separated system. For $0<n_{d} / n<\left(n_{d} / n\right)_{N}$, the system phase separates into a superfluid and a normal state, whereas for $\left(n_{d} / n\right)>\left(n_{d} / n\right)_{N}$ the system is in the normal state. These two different regions are labelled as PS and $\mathrm{N}$ in Fig 2. The dependence of $\left(n_{d} / n\right)_{N}$ on $\gamma$ is a result of competition between the $\gamma$ dependence of $(h / \mu)_{c}$ and that of the density of states. For large $\gamma$, using the asymptotic form for $(h / \mu)_{c}$ discussed before, we get $\left(n_{\downarrow} / n_{\uparrow}\right)_{N} \approx\left(2 / c_{1}\right)^{3 / 2} / \gamma^{3 / 5}$, and thus $\left(n_{d} / n\right)_{N} \approx 1-2^{5 / 2} /\left(c_{1}^{3 / 2} \gamma^{3 / 5}\right)$. This asymptotic dependence is also plotted in Fig 2. The dependence of $\left(n_{\downarrow} / n_{\uparrow}\right)_{N}$ on $\gamma$ is a combined result of $\left(1+(h / \mu)_{c}\right) \propto 1 / \gamma^{3 / 5}$ and the ratio of density of states of the down to up particles $\left(\propto \gamma^{-1}\right)$. This competition also results in the non-monotonic behavior of $\left(n_{d} / n\right)_{N}$ on $\gamma$, with a minimum occuring at $\gamma$ slightly larger than $\gamma_{*}$.

The density of the normal fluid is given by $n_{N}=$ $\frac{1}{6 \pi^{2}}\left[\left(2 m_{\uparrow}(\mu+h)\right)^{3 / 2}+\left(2 m_{\downarrow}(\mu-h)\right)^{3 / 2} \mathcal{H}\right]$ and thus its ratio to that of the superfluid 
state is

$$
\left(\frac{n_{N}}{n_{S}}\right)=\frac{\zeta_{1}^{3 / 2}(0)}{2}\left[\left(\frac{1+\gamma}{2}\right)^{3 / 2}\left(1+\left(\frac{h}{\mu}\right)_{c}\right)^{3 / 2}+\left(\frac{1+\gamma^{-1}}{2}\right)^{3 / 2}\left(1-\left(\frac{h}{\mu}\right)_{c}\right)^{3 / 2} \mathcal{H}\right]
$$

The dependence of $n_{N} / n_{S}$ as a function of $\gamma$ is as shown in Fig 3. $n_{N} / n_{S}$ increases with increasing $\gamma$. For $\gamma<\gamma_{*} \approx 3.9, n_{N} / n_{S}<1$, but for $\gamma>\gamma_{*}, n_{N} / n_{S}>1$. At $\gamma_{*}$ where $(h / \mu)_{c}=0, n_{N} / n_{S}$ is exactly unity as can be verified easily by comparing eqs (10) and (11). For large $\gamma$, we have $n_{N} / n_{S} \sim \zeta_{1}^{3 / 2}(0)\left[c_{1}^{3 / 2} \gamma^{3 / 5}+2^{3 / 2}\right] / 2^{5 / 2}$, hence $n_{N} / n_{S}$ increases with $\gamma$ roughly as $\gamma^{3 / 5}$. This dependence is again a combination of $\gamma$ dependence of $(h / \mu)_{c}$ and the density of states factors. The asymptotic behavior is also plotted in Fig 3 ,

The ratio $n_{N} / n_{S}$ and $\left(n_{d} / n\right)_{N}$ above are directly reflected in the density profile of a trapped gas. Let us consider for simplicity an isotropic harmonic oscillator trap, with trap potential energy for the two species $\frac{1}{2} \alpha_{\sigma} r^{2}$. The local chemical potentials are given by $\mu_{\sigma}(r)=\mu_{\sigma}^{o}-\frac{1}{2} \alpha_{\sigma} r^{2}$, and hence $\mu(r)=\mu^{o}-\frac{1}{4}\left(\alpha_{\uparrow}+\alpha_{\downarrow}\right) r^{2}$ and $h(r)=h^{o}-\frac{1}{4}\left(\alpha_{\uparrow}-\alpha_{\downarrow}\right) r^{2}$. Generally $h(r) / \mu(r)$ increases from the center towards outside. Therefore a superfluid core exists for $r<r_{c}$ where $r_{c}$ is defined by $h\left(r_{c}\right) / \mu\left(r_{c}\right)=(h / \mu)_{c}$. In this region, the local density $n(r)$ is given by $\left(4 m_{r} \mu(r)\right)^{3 / 2} /\left(3 \pi^{2} \zeta_{1}^{3 / 2}(0)\right)$ with $n_{\uparrow}(r)=n_{\downarrow}(r)=n(r) / 2$. For $r>r_{c}$ we have a normal fluid. There $n_{\sigma}(r)=\left[2 m_{\sigma}(\mu(r) \pm h(r))\right]^{3 / 2} /\left(6 \pi^{2}\right)$ provided $\mu(r) \pm h(r)>0$ and vanishes otherwise. As an example, we show in Fig 4 the density profile for the case $\alpha_{\uparrow}=\alpha_{\downarrow}=\alpha, \gamma=6.7\left(\approx m\left({ }^{40} \mathrm{~K}\right) / m\left({ }^{6} \mathrm{Li}\right)\right)$. This figure clearly shows that the density at $r=r_{c}^{+}$is larger than that of $r_{c}^{-}$(with ratio $\approx 1.2$, c.f. Fig 3 also $\left.\left(n_{\downarrow} / n_{\uparrow}\right)\right|_{r_{c}^{+}} \approx 0.12$ corresponding to $\left(n_{d} / n\right)_{N} \approx 0.78$, c.f. Fig 2 2). For comparison, the density profile for the case of equal mass $(\gamma=1)$ is shown in the inset of Fig 4 which shows a density drop from the superfluid core to the normal region. We also note that in our present case, the minority particles have a larger cloud radius than the majority. This is because of the lighter mass of the minority species, so that in fact $\mu_{\downarrow}^{o}>\mu_{\uparrow}^{o}$ and $h^{o}<0$.

In conclusion, we have studied the superfluid pairing between unequal mass Fermions, in particular at resonance. We show that, for equal populations, the thermodynamic properties of the system are the same as the equal mass case except for a simple rescaling, whereas for unequal populations, they depend crucially on the mass ratio of the two species. There is an abundance of stable or long-lived isotopes with very different masses even within the alkalis, ranging from ${ }^{2} \mathrm{H}$ to ${ }^{134} \mathrm{Cs}$, and thus ample opportunities to test the present predications. For example, at resonance, a ${ }^{2} \mathrm{H}$ (majority)- ${ }^{40} \mathrm{~K}$ (minority) mixture would not phase separate, 
whereas a ${ }^{40} \mathrm{~K}$ (majority)- ${ }^{2} \mathrm{H}$ (minority) mixture would. For ${ }^{6} \mathrm{Li}$ (majority)- ${ }^{40} \mathrm{~K}$ (minority) mixture, the system would phase separate with a density drop from the superfluid core to a (completely polarized) normal region, (in contrast to equal mass case, where the normal region is only partially polarized), whereas ${ }^{40} \mathrm{~K}$ (majority) $-{ }^{6} \mathrm{Li}($ minority) mixture would have a density jump.

Very recently, another preprint [14] appears which also deals with unequal mass Fermions. This research was supported by the NSC of Taiwan under grant numbers NSC94-2112-M194-008 (STW), NSC94-2112-M-194-001 (CHP), and NSC94-2112-M-001-002 (SKY).

[1] W. C. Stwalley, Phys. Rev. Lett. 37, 1628 (1976), E. Tiesinga, B. J. Verhaar, H. T. C. Stoof, Phys. Rev. A 47, 4114 (1993)

[2] D. M. Eagles, Phys. Rev. 186, 456 (1969); A. J. Leggett, in Modern Trends in the theory of condensed matter, edited by A. Pekalski and J. Przystawa (Springer-Verlag, Berlin, 1980); J. R. Engelbrecht et al, Phys. Rev. B 55, 15153 (1997)

[3] C. A. Regal, M. Greiner, and D.S. Jin, Phys. Rev. Lett. 92, 040403 (2004); T. Bourdel et al, ibid. 93, 050401 (2004); C. Chin et al., Science 305, 1128 (2004); J. Kinast et al., ibid. 307, 1296 (2005); M. W. Zwierlein et al, Nature (London) 435, 1047 (2005); and references therein.

[4] Y. Ohashi and A. Griffin, Phys. Rev. A 67, 063612 (2003); J. Carlson et al., Phys. Rev. Lett. 91, 050401 (2003); A. Perali et al., ibid, 92, 220404 (2004); G. Astrakharchik et al, ibid, 93, 200404 (2004); Q. Chen et al, Phys. Rep. 412, 1 (2005); A. Bulgac et al., ibid, 96, 090404 (2006); and references therein.

[5] J. Carlson and S. Reddy, Phys. Rev. Lett. 95, 060401 (2005); C.-H. Pao, S.-T. Wu and S.-K. Yip, Phys. Rev. B 73, 132506 (2006); D. T. Son and M. A. Stephanov, cond-mat/0507586, D. E. Sheehy and L. Radzihovsky, Phys. Rev. Lett. 96, 060401 (2006)

[6] M. W. Zwierlein et al, Science 311, 492 (2006); G. B. Partridge et al, ibid, 311, 503 (2006).

[7] W. Yi and L.-M. Duan, Phys. Rev. A 73, 031604 (2006); T. N. De Silva and E. J. Mueller, ibid, 051602 (2006); F. Chevy, Phys. Rev. Lett., 96, 130401 (2006); M. Haque and H. T. C. Stoof, cond-mat/0601321; C.-H. Pao and S.-K. Yip, J. Phys. Cond.Matt. 18, 5567 (2006)

[8] C. A. Stan et al, Phys. Rev. Lett. 93, 143001 (2004); S. Inouye et al, ibid, 93, 183201 (2004); both these references are for Feshbach resonances between a boson and a fermion, but we see 
no reason which forbids a resonance between two different fermionic atoms.

[9] M. M. Forbes et al, Phys. Rev. Lett. 94, 017001 (2005); M. Alford, ibid, 92, 222001 (2004); and references therein.

[10] Explicitly, this free energy is $\Omega(\Delta, \mu, h)=\sum_{\vec{k}}\left[\frac{\hbar^{2} k^{2}}{4 m_{r}}-\mu-\tilde{E}(k)+\frac{2 m_{r}}{\hbar^{2} k^{2}}|\Delta|^{2}+E_{\uparrow}(k) f\left(E_{\uparrow}(k)\right)\right]-$ $\frac{m_{r}}{2 \pi \hbar^{2} a}|\Delta|^{2}$ where $f$ is the Fermi function, and $E_{\sigma}(k), \tilde{E}(k), \mu$ are defined in text.

[11] S.-T. Wu and S.-K. Yip, Phys. Rev. A 67, 053603 (2003).

[12] G. Sarma, J. Phys. Chem. Solids, 24, 1029 (1963)

[13] A. Sedrakian et al, Phys. Rev. A 72, 013601 (2005); P. Fulde and R. A. Ferrell, Phys. Rev. 135, A550 (1964); A. I. Larkin and Yu. N. Ovchinnikov, Zh. Éksp. Teor. Fiz. 47, 1136 (1964) [Sov. Phys. JETP 20, 762 (1965)]

[14] M. Iskin and C. A. R. Sá de Melo, cond-mat/0604184 


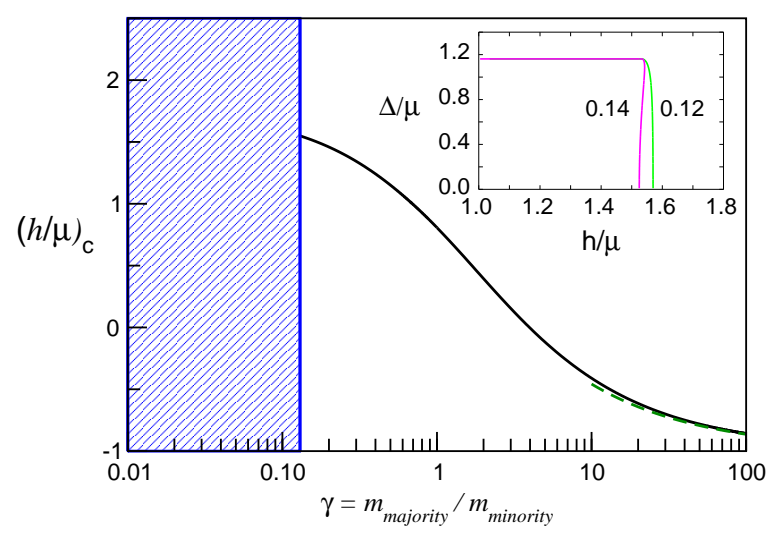

FIG. 1: (color online) $(h / \mu)_{c}$ as a function of $\gamma$ for $\gamma>\gamma_{H}$. The dashed line is the asymptotic formula in text. Inset: $\Delta / \mu$ as a function of $h / \mu$ for $\gamma=0.14$ and $\gamma=0.12$.

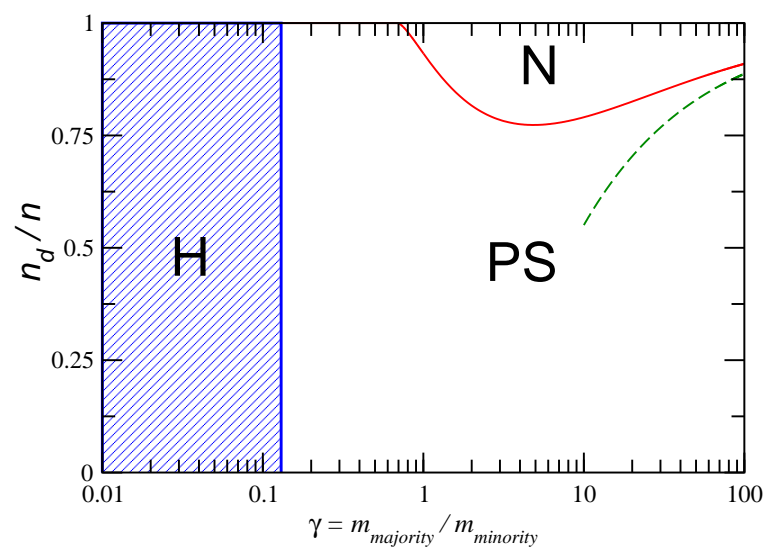

FIG. 2: (color online) The bulk phase diagram at resonance. For $n_{d} / n=0$, the system is a superfluid, whereas it is normal for $n_{d} / n=1$. For $0<n_{d} / n<1$, the phases are: $\mathrm{H}$ - the homogeneous phase, PS - phase separated state, N - normal phase. The dashed line is the asymptotic formula discussed in text. 


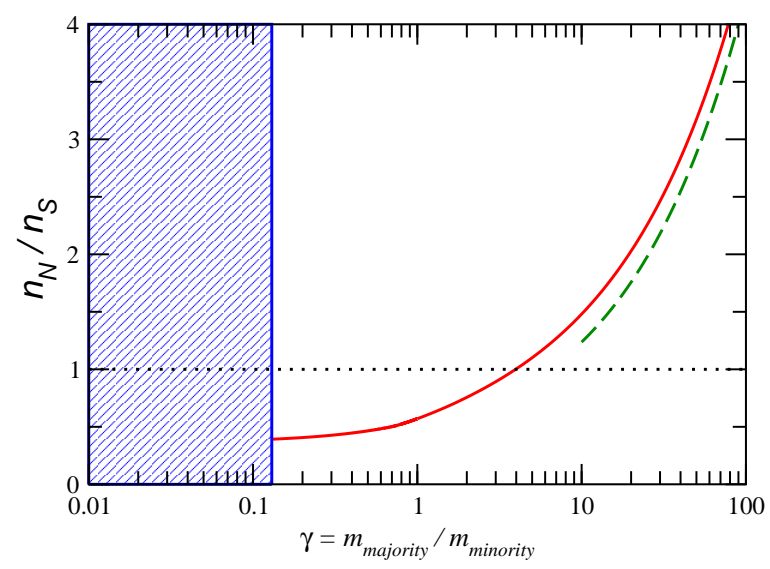

FIG. 3: (color online) The ratio of $n_{N}$ to $n_{S}$ at phase equilibrium for the phase separated region. The dashed line is the asymptotic formula discussed in text.

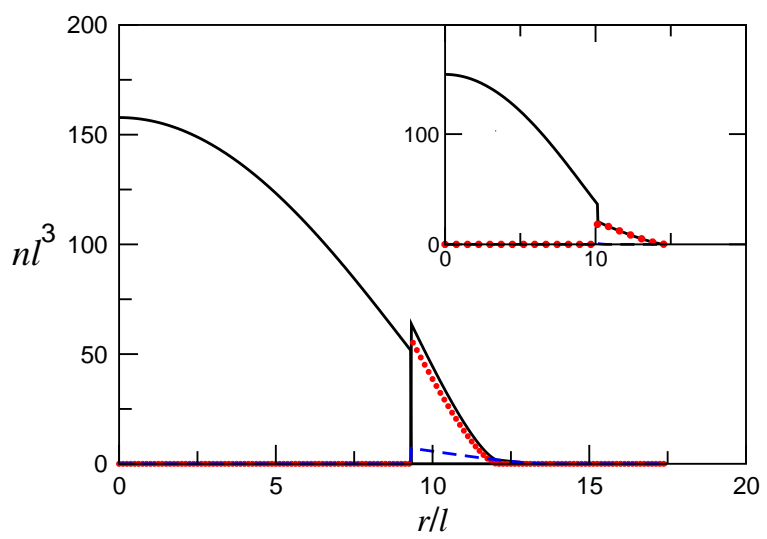

FIG. 4: (color online) Density profile at resonance for $N=4.1 \times 10^{5}, N_{d}=6.6 \times 10^{4}, N_{d} / N=0.16$. $\alpha_{\uparrow}=\alpha_{\downarrow}=\alpha, \gamma=6.7$. Full line: total density, dots and dashed: $n_{\uparrow,(\downarrow)}$ in the normal fluid region. Here $r$ is normalized to $l \equiv 1 /\left[\alpha\left(2 m_{r}\right)\right]^{1 / 4}$ and the density $n$ to $l^{-3}$. Inset: density profile for the same $N$ and $N_{d}$ for $\gamma=1 . n_{\downarrow}(r)$ for the normal phase is very small and thus only barely visible. The density profiles with the same $N_{d} / N$ but $N_{\text {new }}=\beta N$ can be obtained by the scaling relation $n_{\text {new }}\left(\beta^{1 / 6} r\right)=\beta^{1 / 2} n(r)$. 